\title{
A Comparison of Heritability Estimates by Classical Twin Modeling and Based on Genome-Wide Genetic Relatedness for Cardiac Conduction Traits
}

\author{
Ilja M. Nolte,, ${ }^{1, *}$ Joeri A. Jansweijer, ${ }^{2, *}$ Hariette Riese, ${ }^{1,3}$ Folkert W. Asselbergs, ${ }^{4}$ Pim van der Harst, ${ }^{5}$ \\ Timothy D. Spector, ${ }^{6}$ Yigal M. Pinto, ${ }^{2}$ Harold Snieder, ${ }^{1}$ and Yalda Jamshidi ${ }^{7}$ \\ ${ }^{1}$ Department of Epidemiology, University of Groningen, University Medical Center Groningen, Groningen, \\ The Netherlands \\ ${ }^{2}$ Department of Cardiology, University of Amsterdam, Academic Medical Center, Amsterdam, The Netherlands \\ ${ }^{3}$ Department of Psychiatry, Interdisciplinary Center Psychopathology and Emotion regulation (ICPE), \\ University of Groningen, University Medical Center Groningen, Groningen, The Netherlands \\ ${ }^{4}$ Department of Cardiology, Division of Heart and Lungs, University Medical Center Utrecht, Utrecht, The Netherlands \\ ${ }^{5}$ Department of Cardiology, University of Groningen, University Medical Center Groningen, Groningen, The Netherlands \\ ${ }^{6}$ Department of Twin Research and Genetic Epidemiology, King's College London, London, UK \\ ${ }^{7}$ Genetics Unit, Cardiovascular and Cell Sciences Institute, St George's University of London, London, UK
}

\begin{abstract}
Twin studies have found that $\sim 50 \%$ of variance in electrocardiogram (ECG) traits can be explained by genetic factors. However, genetic variants identified through genome-wide association studies explain less than $10 \%$ of the total trait variability. Some have argued that the equal environment assumption for the classical twin model might be invalid, resulting in inflated narrow-sense heritability $\left(h^{2}\right)$ estimates, thus explaining part of the 'missing $h^{2}$ '. Genomic relatedness restricted maximum likelihood (GREML) estimation overcomes this issue. This method uses both family data and genome-wide coverage of common SNPs to determine the degree of relatedness between individuals to estimate both $h^{2}$ explained by common SNPs and total $h^{2}$. The aim of the current study is to characterize more reliably than previously possible ECG trait $h^{2}$ using GREML estimation, and to compare these outcomes to those of the classical twin model. We analyzed ECG traits (heart rate, PR interval, QRS duration, RV5+SV1, QTc interval, Sokolow-Lyon product, and Cornell product) in up to 3,133 twins from the TwinsUK cohort and derived $h^{2}$ estimates by both methods. GREML yielded $h^{2}$ estimates between $47 \%$ and $68 \%$. Classical twin modeling provided similar $h^{2}$ estimates, except for the Cornell product, for which the best fit included no genetic factors. We found no evidence that the classical twin model leads to inflated $h^{2}$ estimates. Therefore, our study confirms the validity of the equal environment assumption for monozygotic and dizygotic twins and supports the robust basis for future studies exploring genetic variants responsible for the variance of ECG traits.
\end{abstract}

Keywords: genetics, arrhythmia, heritability, twin study

The 12-lead electrocardiogram (ECG) provides diagnostic and prognostic information regarding cardiac morbidity and mortality in both patients with heart disease (Algra et al., 1991; Copie et al., 1996; Dekker et al., 1994; Murkofsky et al., 1998) and healthy subjects (Dekker et al., 2004; Schouten et al., 1991). ECG measurements are complex traits with multiple genetic and environmental determinants and are known to be influenced by both age (Yasumura \& Shibata, 1989) and gender (Storstein et al., 1991). Genetic contributions to the ECG traits PR interval, QT interval, QRS duration, and RR interval have been investigated in twin and family studies (Dalageorgou et al.,
2008; Hanson et al., 1989; Havlik et al., 1980; Hong et al., 2001; Li et al., 2009; Mathers et al., 1961; Mutikainen et al., 2009; Russell et al., 1998; Singh et al., 1999; Voss et al., 1996).

ReCeived 22 August 2017; ACCEPTed 4 September 2017. First published online 17 October 2017.

ADDRESS FOR CORRESPONDENCE: Ilja M. Nolte, Department of Epidemiology (FA40), University Medical Center Groningen, P.O. Box 30.001, 9700 RB Groningen, The Netherlands. E-mail: i.m.nolte@umcg.nl

${ }^{*}$ These authors contributed equally to this work. 
Typically, 35-55\% of PR, QT, and RR interval variability in the population is accounted by genetic variation (Arking et al., 2006; Chambers et al., 2010; Chang et al., 2008; Dalageorgou et al., 2008; George et al., 2009; Hanson et al., 1989; Havlik et al., 1980; Holm et al., 2010; Hong et al., 2001; Li et al., 2009; Newton-Cheh et al., 2007; Nolte et al., 2009; Pfeufer et al., 2010; Russell et al., 1998; Singh et al., 1999; Smith et al., 2009). Heritability $\left(h^{2}\right)$ for QRS duration and voltage criteria also appears to be within this range (Hanson et al., 1989; Li et al., 2009; Mutikainen et al., 2009), although a number of studies have failed to show evidence of significant $h^{2}$ (Havlik et al., 1980; Russell et al., 1998; Smith et al., 2009).

The extent to which genetic variants can be expected to account for ECG trait variability is an important factor guiding gene-finding studies. Genome-wide association studies have successfully identified many variants associated with ECG traits (Arking et al., 2006; Chambers et al., 2010; Holm et al., 2010; Newton-Cheh et al. 2007; NewtonCheh et al., 2009; Nolte et al., 2009; Pfeufer et al., 2009; Pfeufer et al., 2010). However, the variants discovered to date explain less than $10 \%$ of the total variability, which is far below the $\mathrm{h}^{2}$ estimates in the earlier-mentioned twin and family studies. This 'missing heritability' may in part be explained by the stringent genome-wide significance threshold used to detect associated variants. Thus, many common variants with smaller effect sizes, as well as rare or lowfrequency variants may still need to be identified. On the other hand, it has also led some to argue that the equal environment assumption of the classical twin model stating that monozygotic (MZ) and dizygotic (DZ) twins share their environments to the same extent may be invalid, resulting in inflated $\mathrm{h}^{2}$ estimates (Maher, 2008; Turkheimer, 2011; Zuk et al., 2012).

Heritability can be estimated based on the comparison of trait correlations between relatives of varying degrees. The classical twin model quantifies the genetic contribution to a trait by relating the assumed difference in genetic similarity between groups of $\mathrm{MZ}$ and $\mathrm{DZ}$ twin pairs to the difference in trait correlations. MZ twins are formed from the splitting of a single embryo and are genetically identical, whereas DZ twins are the product of separate fertilizations and are as genetically similar as other siblings. The classical twin model therefore uses the assumption that DZ twins, like other siblings, share $50 \%$ of their segregating genes. If genetic variation defines the trait under study, the correlation for MZ twin pairs is expected to be larger than for $\mathrm{DZ}$ twin pairs.

Zaitlen et al. (2013) elaborated on a method devised by Yang et al. (2010), resulting in a genomic relatedness restricted maximum likelihood (GREML) variance components method that estimates $h^{2}$ both from identity-bydescent (IBD) and identity-by-state (IBS) sharing of alleles between related and unrelated individuals. The sum of these $h^{2}$ values yields a more accurate estimate of the total narrow-sense $h^{2}$ because no assumptions are made on the extent of genetic similarity, dominance effects, and epistasis (Zaitlen et al., 2013).

In this study, we determined $h^{2}$ estimates using both the classical twin model and GREML for all major ECG traits in a large sample of female twins. By comparing both methods in the same cohort, we aim to establish whether the classical twin model provides inflated heritability estimates and hence whether this could explain part of the missing heritability. In addition, we performed a simulation study to assess the effect of increased environmental sharing for MZ compared to $\mathrm{DZ}$ twins on the heritability estimates by both methods.

\section{Material and Methods}

\section{Subjects}

Data were obtained from the TwinsUK cohort, which is a large cohort of twin pairs that comprises unselected, mostly female volunteers ascertained from the general population through national media campaigns in the United Kingdom. Means and ranges of quantitative phenotypes in TwinsUK were similar to an age-matched singleton sample from the general population (Andrew et al., 2001). Zygosity was determined by a standardized questionnaire and confirmed by DNA fingerprinting. Demographic and phenotype data were obtained via nurse-administered questionnaires. All TwinsUK studies have ethical approval from the Guy's and St Thomas' Ethics Committee (Moayyeri et al., 2013).

\section{Electrocardiogram Measures}

ECGs were recorded using the Cardiofax ECG-9020K (Nihon Kohden UK Ltd., Middlesex, UK) or the Philips Page Writer Trim 1 (Philips Medical Systems, Andover, USA). ECG data from 3,313 Caucasian women (of whom 3,034 were part of complete twin pairs and 279 of incomplete twin pairs) were used in the current analyses. The following ECG traits were measured: heart rate, $\mathrm{PR}$, and QT interval, QRS duration, and three voltage-related traits: (1) RV5+SV1 = $\mathrm{R}$ in V5 + S in V1; (2) Sokolow-Lyon product $=(\mathrm{R}$ in V5 or V6, whichever is higher, $+\mathrm{S}$ in V1) $\times$ QRS duration; (3) Cornell product $=(\mathrm{R}$ in $\mathrm{aVL}+\mathrm{S}$ in $\mathrm{V} 3$ [ +6 in women] $) \times$ QRS duration) (Calderón et al., 2010).

The Cardiofax ECG-9020K (Nihon Kohden UK Ltd., Middlesex, UK) also produced automatically scored diagnostic ratings of the ECGs (ECG codes). These were used to divide the subjects in cases and controls for various binary traits. For 911 (27.5\%) subjects diagnostic ratings of the ECGs were not available, leaving 2,402 Caucasian women for categorization into not-affected versus affected.

\section{Genotyping and Imputation}

Twins were genotyped using either the HumanHap300, the HumanHap610Q, the HumanHap1M Duo, or the HumanHap1.2M Duo 1M array (Illumina, San Diego, USA). For each MZ twin pair, only one of the twins was genotyped. 
The quality of the genotype data was checked per chip with PLINK (Purcell et al., 2007). SNPs with a minor allele frequency $<1 \%$, genotype call rate $<95 \%$, or a $\operatorname{HWE} p$ value $<10^{-5}$ were excluded. Samples with a call rate $<95 \%$, those whose sex did not match the database, were non-Caucasian, or showed moderate genetic relation to a large number of other individuals were also excluded (Nolte et al., 2009). The remaining genotype data were merged and then imputed using HapMap Phase III (release 2, build 36) as a reference set (Howie et al., 2009). This reference set was chosen because the HapMap III SNP set was optimized to capture common genetic variation in the human genome (International HapMap 3 Consortium, 2010), which is required for the GREML analyses is described later. The resulting imputed data sets were next converted to best guess genotypes where only genotypes with a probability $>0.9$ for one of the three genotypes were assigned and otherwise it was set to missing. SNPs with a minor allele frequency $<5 \%$, an imputation quality $<0.5$, or a call rate $<0.95$ were removed. For the MZ twin pairs the genotypes of nongenotyped twin were assumed to be identical to those of the genotyped co-twin and added as such to the database. Both genome-wide and ECG data were available for 2,943 twins.

\section{Statistical Analyses}

MZ and DZ twins were compared for quantitative demographic and ECG variables using a Student's $t$ test or MannWhitney $U$ test depending on the distribution of the variable. Binary traits were compared between MZ and DZ twins using chi-square test or Fisher's exact test, whichever was appropriate. For the heritability analyses of the continuous ECG parameters, data on the ECG traits of 180 (out of $3,313=5.4 \%)$ women were excluded because of (1) use of anti-arrhythmic drugs $(n=6),(2)$ presence of a heart condition (e.g., ischemic disease, stroke or bypass surgery, $n=$ 73), (3) PR-interval was missing (atrial fibrillation, $n=53$ ), and (4) QRS duration $>120 \mathrm{~ms}(n=48)$. For the classical twin analyses, co-twins of these women were included if available, as Mx can handle data of incomplete twin pairs.

Classical twin modeling. In the classical twin model the differences between $\mathrm{MZ}$ and DZ (co)variances on a trait are used to investigate the relative contribution of genetic and environmental influences to individual differences in a trait. The observed (co)variances of twin data are expressed as a function of latent genetic and environmental factors. The variance components considered are additive genetic variation (A), which reflects narrow-sense heritability $\left(h^{2}\right)$, shared environmental variation $(\mathrm{C})$, and a unique environmental component $(\mathrm{E})$ that is not shared by family members and includes measurement error. For (MZ and DZ) twins reared together $\mathrm{C}$ is assumed to correlate perfectly, whereas A correlates 1 in $\mathrm{MZ}$ twins and 0.5 in $\mathrm{DZ}$ twins since $\mathrm{MZ}$ twins share all their segregating genes and DZ pairs share $50 \%$ on average. The total variance of a trait for each in- dividual is the sum of $\mathrm{A}, \mathrm{C}$, and $\mathrm{E}$. The effect and significance of these factors are inferred by fitting the observed (co)variances of the data for $\mathrm{MZ}$ and $\mathrm{DZ}$ pairs to their predicted (co)variances of the hypothesized model (ACE, $\mathrm{AE}, \mathrm{CE}$, or E). The structural equation modeling program Mx was used to estimate model parameters by minimizing a goodness-of-fit statistic between observed and predicted (co)variances (Neale et al., 2003).

Heritability estimates using the genomic relationship matrix. To evade assumptions on genetic similarity and reduce confounding with non-genetic factors, we also used GREML analyses in the software package Genome-wide Complex Trait Analysis (GCTA; Yang et al., 2011) to estimate $h^{2}$ both from IBD and IBS sharing of alleles between related and unrelated individuals using a genetic relationship matrix. The degree of relatedness between individuals was quantified using genotyped common SNPs. Taking into account only the unrelated individuals, this method yields an estimate of heritability explained by common SNPs $\left(h^{2}{ }_{\text {SNP }}\right)$ (Visscher et al., 2006; Yang et al., 2010). However, in the closely related individuals (proportion of IBS alleles $>0.05$ ), it yields an estimate of heritability explained by other additive genetic factors not measured by the genotyped SNPs $\left(h^{2}{ }_{\text {IBS }>0.05}\right)$, based on the assumption that IBS then represents IBD. Consequently, GREML does not assume that DZ twins share exactly $50 \%$ of their genome, but uses the true sharing, which ranges from $35 \%$ to $65 \%$. The sum of joint estimates of $h^{2}$ SNP and $h^{2}$ IBS $>0.05$ is equal to the narrow-sense heritability $h^{2}$ (Zaitlen et al., 2013). We used residuals of the resting ECG parameters adjusted for age and ECG machine, and added five principal components calculated with GCTA as covariates to the GREML analyses.

To assess the putative effect of unequal environments between MZ and DZ twins, GREML estimates were calculated for the complete data set using both MZ twins, as well as for the data set with only the single genotyped $M Z$ twin included (-grm-cutoff $<0.95 ; n=2,912)$. In the latter case, the phenotype values of the MZ twin were taken as the average of the values of both $\mathrm{MZ}$ twins in order to reduce noise and optimize the sample size, because for some of the genotyped MZ twins no ECG data for one twin were available, but for her co-twin they were.

For comparison with the joint GREML heritability estimates, we also applied GREML to only the unrelated individuals (relatedness $<0.05 ; n=1.390$ ) using a single genetic relationship matrix, obtaining another unbiased estimate of $h^{2}$ SNP (Yang et al., 2010), and to all individuals with a single matrix in which genetic relatedness $<0.05$ was set to zero, this analysis provides another unbiased estimate of the narrow-sense $h^{2}$ (Zaitlen et al., 2013). In addition, a recently proposed improved GREML analysis was applied to the unrelated individuals, which uses a sliding window approach to fit the region-specific linkage disequilibrium 
heterogeneity across the genome and accounts for differences in minor allele frequencies (GREML-LDMS) (Yang et al., 2015). A linkage disequilibrium $r^{2}$ threshold of 0.01 was used in order to avoid chance correlations between SNPs that were not in linkage disequilibrium. SNPs were stratified to two minor allele frequency bins $(<0.2$ and $0.2-$ 0.5 ) and two linkage disequilibrium bins (based on the linkage disequilibrium median). Note that the lower allele frequency bin hardly contains SNPs with minor allele frequencies below $5 \%$, and hence we could not determine the heritability explained by low frequency and rare SNPs.

BMI and height. For comparative reasons, all modelings were also conducted on BMI and height.

Simulation study. In order to assess the effect of violation of the equal environment assumption for $\mathrm{MZ}$ and $\mathrm{DZ}$ twins on the Mx and GREML results, we simulated 5,000 data sets with extreme phenotypes for the $\mathrm{MZ}$ and $\mathrm{DZ}$ twins in our data set using the observed genetic relations between the twins, $\mathrm{A}=0.45, \mathrm{C}=0.2$, with environmental correlation $=1$ for $M Z$ twin pairs and 0 for DZ twin pairs. With these settings, the MZ twin correlation was 0.65 and the DZ twin correlation was 0.225 , from which the narrow-sense $h^{2}$ would be estimated to be 0.65 . For comparison we also performed simulations using no environmental factor (i.e., $C=0$ ) for both $\mathrm{MZ}$ and $\mathrm{DZ}$ twin pairs (resulting in $\mathrm{MZ}$ twin correlation of 0.45 , a DZ twin correlation of 0.225 , and a $h^{2}$ of 0.45$)$. Mx was applied to each of the 5,000 data sets, as well as GREML using: (1) the complete data sets of MZ and DZ twin pairs plus singletons and (2) only the DZ twins pairs, the singletons, and one of the MZ twins.

\section{Results}

The general characteristics, medication use, and resting ECG values are presented in Table 1. MZ twins were significantly older than the DZ twins (53.9 vs. 50.9 years). Medication use was mostly comparable, but DZ twins more often used thiazides and beta-blockers. Mean values for QRS duration, and QT interval-both uncorrected (QT) and corrected for heart rate (QTc) using the Framingham or Bazett's formula, differed significantly between MZ and DZ twins. The differences in QRS duration remained significant after correction for age and ECG machine, but for QTc the differences became non-significant after these corrections. No differences were observed between $\mathrm{MZ}$ and DZ twins for the diagnostic ECG categories (Supplementary Table S1).

In Supplementary Table S2, the correlations among the ECG traits and with age are provided. As expected, heart rate correlated highly with QT interval. Age was significantly correlated with all ECG variables, and correction for age and ECG machine led to a decrease in the correlations of QT interval with $\mathrm{PR}$ interval and heart rate (correlations both no longer significant). As expected, after adjustment, Sokolow-Lyon showed strong correlation with RV5+SV1 (0.87), and the QRS related traits Cornell and Sokolow-Lyon demonstrated the strongest correlations with QRS (0.37 Cornell, 0.30 Sokolow-Lyon).

\section{Simulation Study}

Heritability estimates by both Mx and GREML using the complete data set were biased when the equal environment assumption for MZ and DZ twins was violated $\left(\mathrm{Mx}: h^{2}=\right.$ $0.65 \pm 0.01$; GREML, including both MZ twins: $h^{2}=0.67 \pm$ $0.04)$. However, GREML applied to a data set in which only one of the MZ twins was included, provided unbiased estimates $\left(h^{2}=0.47 \pm 0.05\right)$. When no environmental sharing for both $\mathrm{MZ}$ and DZ twin pairs was simulated and the equal environment assumption holds, all estimates were unbiased (Mx: $h^{2}=0.44 \pm 0.03$; GREML, including both MZ twins: $h^{2}=0.48 \pm 0.05$; GREML with only one of the MZ twins: $\left.h^{2}=0.47 \pm 0.05\right)$.

\section{Classical Twin Modeling}

Twin correlations were calculated for $\mathrm{MZ}$ and $\mathrm{DZ}$ twin pairs separately and suggested that all ECG traits were heritable, except the Cornell product (Table 2; Supplementary Table S3; Figure 1). Indeed, quantitative genetic analysis showed that for almost all studied ECG variables, the AE model including only additive genetic (A) and unique environmental (E) variance components fitted the data best compared to the full ACE model. Estimates of $h^{2}$ for these traits ranged from 0.53 to 0.61 and were statistically significant. The only exception was the Cornell product, where the CE model including only shared environmental (C) and E variance components (Table 2) fitted the data best.

\section{Heritability Estimates Using the Genomic Relationship Matrix}

Using genomic data of all MZ and DZ twin pairs, joint estimation by GREML yielded total narrow-sense $h^{2}$ estimates for all ECG traits ranging from 0.47 to 0.68 , including the Cornell Product (Table 3; Figure 1). These proportions of explained variance are similar to the $h^{2}$ estimates obtained from classical twin modeling, except for the Cornell product. Similar estimates of $h^{2}$ were obtained by the GREML single matrix analysis (Supplementary Figure $\mathrm{S} 1$ ). When only the single genotyped MZ twin was used, the estimates of $h^{2}$ did not decrease significantly, indicating that the equal environment assumption holds (Figure 2). Note that for the Cornell product and for height the $h^{2}$ estimate even increased significantly $(p<.05)$.

The $h_{\text {SNP }}^{2}$ estimates were significant for heart rate $\left(h^{2}\right.$ SNP $=0.24,95 \% \mathrm{CI}[0.02,0.46]), \mathrm{PR}$ interval $\left(h^{2}{ }_{\mathrm{SNP}}=0.24,95 \%\right.$ CI $[0.02,0.47])$, QRS duration $\left(h^{2}{ }_{\text {SNP }}=0.26,95 \%\right.$ CI [0.04, $0.48])$, and QTc interval $\left(h_{\mathrm{SNP}}^{2}=0.30,95 \% \mathrm{CI}[0.08,0.52]\right)$. Due to the smaller sample sizes, no reliable $h_{\text {SNP }}^{2}$ estimates could be obtained for RV5+SV1, Sokolow-Lyon Product, 


\section{TABLE 1}

Subject Characteristics for MZ and DZ Twins Separately for the ECG Parameters Based on Maximally 3,133 twins After Exclusion of the 180 Women Who Met Our Exclusion Criteria for the Heritability Analyses

\begin{tabular}{|c|c|c|c|}
\hline & $M Z(n=1,320)$ & $\mathrm{DZ}(n=1,813)$ & $p$ value (corrected $p$ value) \\
\hline Age (years) & $53.9(12.73)$ & $50.9(12.42)$ & $<.001$ (NA) \\
\hline $\mathrm{BMI}\left(\mathrm{kg} / \mathrm{m}^{2}\right)^{\mathrm{b}}$ & 25.5 (4.39) & $25.6(4.65)$ & $n s(n s)$ \\
\hline Height $(\mathrm{cm})$ & $161.9(5.50)$ & $162.6(6.08)$ & $.004(n s)$ \\
\hline \multicolumn{4}{|l|}{ Medication (\% users) } \\
\hline - Thiazides & 1.5 & 3.5 & $<.001[<0.001]$ \\
\hline - Hypnotics or sedatives & 1.2 & 1.2 & $n s(\mathrm{NA})$ \\
\hline - Anticonvulsants & 0.5 & 0.5 & $n s(\mathrm{NA})$ \\
\hline - Antipsychotics & 0.3 & 0.2 & $n s(N A)$ \\
\hline - Antibiotics & 0.5 & 0.8 & $n s(N A)$ \\
\hline - Betablockers & 2.3 & 3.2 & $n s(0.027)$ \\
\hline - Antidepressant & 2.9 & 4.2 & $n s(n s)$ \\
\hline Heart rate (bpm) & $66.9(10.38)$ & $66.9(10.26)$ & $n s(n s)$ \\
\hline RR interval $(\mathrm{ms})^{\mathrm{c}}$ & $919.0(141.55)$ & $918.0(141.17)$ & $n s(n s)$ \\
\hline QRS duration (ms) & $87.9(8.14)$ & $86.8(8.18)$ & $<.001[0.03]$ \\
\hline PR interval (ms) & $158.0(22.30)$ & $158.0(22.49)$ & $n s(n s)$ \\
\hline QT interval (ms) & $405.6(27.35)$ & $402.2(27.51)$ & .001 (ns) \\
\hline QTc Framingham $(\mathrm{ms})^{d}$ & $418.1(17.26)$ & $414.9(16.90)$ & $<.001$ (ns) \\
\hline QTc Bazett $(\mathrm{ms})^{\mathrm{e}}$ & $425.2(20.49)$ & $421.8(20.01)$ & $<.001$ (ns) \\
\hline $\mathrm{RV} 5+\mathrm{SV} 1^{\mathrm{f}}$ & $2.2(0.60)$ & $2.2(0.63)$ & $n s(n s)$ \\
\hline Sokolow-Lyon product ${ }^{\mathrm{g}}$ & $1,894.2(570.42)$ & $1,911.2(575.50)$ & $n s(n s)$ \\
\hline Cornell product ${ }^{\mathrm{h}}$ & $881.1(425.20)$ & $859.3(416.25)$ & $n s(n s)$ \\
\hline
\end{tabular}

Note: $\mathrm{BMI}=$ body mass index; $\mathrm{NA}=$ not applicable: $n s=$ not significant, $p$ value $\geq .05$ ${ }^{a} p$ value corrected for age and ECG machine; ${ }^{b} n_{M Z}=998, n_{D Z}=1,734 ;{ }^{c} R R$-interval $=(60 /$ heart rate $)^{\star} 1,000(\mathrm{~ms}) ;{ }^{\mathrm{d}}$ The Framingham Heart Study formula: $\mathrm{QTc}=\mathrm{QT}+0.154^{\star}(1,000-\mathrm{RR}) .{ }^{\mathrm{e}}$ The Bazett formula: QTc $=\mathrm{QT} / \mathrm{RR}^{1 / 2} ;{ }^{f} \mathrm{n}_{\mathrm{MZ}}=778, \mathrm{n}_{\mathrm{DZ}}=1375 ;{ }^{9} \mathrm{n}_{\mathrm{MZ}}=911, \mathrm{n}_{\mathrm{DZ}}=1520 ;{ }^{\mathrm{h}} \mathrm{n}_{\mathrm{MZ}}=$ 911, $\mathrm{n}_{\mathrm{DZ}}=1505$

TABLE 2

Standardized Estimates and 95\% Cls of Genetic and Environmental Influences on Resting ECG Variables Corrected for Age and ECG Machine, Based on the Best-Fitting Variance Components Model

\begin{tabular}{|c|c|c|c|}
\hline & A & C & $\mathrm{E}$ \\
\hline Heart rate & $0.53[0.48,0.58]$ & - & $0.47[0.42,0.52]$ \\
\hline PR interval & $0.55[0.50,0.60]$ & - & $0.45[0.40,0.51]$ \\
\hline QRS duration & $0.54[0.48,0.58]$ & - & $0.46[0.42,0.52]$ \\
\hline QTc interval ${ }^{a}$ & $0.61[0.56,0.65]$ & - & $0.39[0.35,0.44]$ \\
\hline $\mathrm{RV} 5+\mathrm{SV} 1$ & $0.61[0.54,0.66]$ & - & $0.39[0.34,0.46]$ \\
\hline Sokolow-Lyon product & $0.57[0.50,0.64]$ & - & $0.43[0.36,0.50]$ \\
\hline Cornell product (ACE model) & $0.13[0.00,0.37]$ & $0.27[0.10,0.40]$ & $0.61[0.51,0.70]$ \\
\hline Cornell product (best fit) ${ }^{b}$ & - & $0.35[0.29,0.40]$ & $0.65[0.60,0.71]$ \\
\hline $\mathrm{BMI}^{\mathrm{c}}$ & $0.77[0.73,0.80]$ & - & $0.23[0.20,0.27]$ \\
\hline Height & $0.74[0.65,0.83]$ & $0.14[0.05,0.23]$ & $0.12[0.11,0.14]$ \\
\hline
\end{tabular}

and Cornell Product. The GREML estimates applied to unrelated individuals using a single matrix only yielded significant estimates for heart rate $\left(h^{2}{ }_{\text {SNP }} 0.42,95 \%\right.$ CI [0.03, $0.81])$ and QRS duration $\left(h^{2}\right.$ SNP $0.42,95 \%$ CI $\left.[0.04,0.81]\right)$ (Supplementary Figure S1A). These estimates were higher than those obtained by joint modeling, but the standard error was much larger. In addition for the recently proposed GREML-LDMS method applied to the unrelated individuals only the $h^{2}$ SNP estimates for QRS duration $\left(h^{2}{ }_{\mathrm{SNP}}=0.49\right.$, $95 \% \mathrm{CI}[0.05,0.94])$ and height $\left(h^{2}{ }_{\mathrm{SNP}}=0.55,95 \% \mathrm{CI}[0.13\right.$, 0.96]) were significant (Supplementary Figure S1B).

\section{Discussion}

In this study, we showed that both the classical twin model and GREML analysis yielded narrow-sense heritability estimates between $47 \%$ and $68 \%$ for a wide range of ECG traits in a general population cohort of more than 3,000 MZ and DZ twins. The Cornell product was the only trait with discordant results, since the best fitting classical twin model included no additive genetic factors, whereas the GREML heritability estimate was $47 \%$. Our general results for these ECG traits are in line with those from a recent meta-analysis of all twin studies, which reported that the results of $95 \%$ of 
TABLE 3

Joint Estimates of the Narrow-Sense and Common SNP Heritability by GREML Applied to the Complete Data Set $(n=2,943)$ and to the Data Set With Only the Single Genotyped MZ Twins $(n=2,411)$

\begin{tabular}{|c|c|c|c|c|}
\hline & $h^{2}+[95 \% \mathrm{Cl}]$ both $\mathrm{MZ}$ twins & $h^{2}+[95 \% \mathrm{Cl}]$ one $\mathrm{MZ}$ twin & $h^{2} \mathrm{sNP}+[95 \% \mathrm{Cl}]$ both $\mathrm{MZ}$ twins & $h^{2} \mathrm{SNP}+[95 \% \mathrm{Cl}]$ one $\mathrm{MZ}$ twin \\
\hline Heart rate & $0.53[0.48,0.59]$ & $0.45[0.31,0.58]$ & $0.24[0.02,0.46]$ & $0.26[0.03,0.49]$ \\
\hline PR interval & $0.55[0.50,0.60]$ & $0.54[0.41,0.67]$ & $0.24[0.02,0.47]$ & $0.26[0.02,0.50]$ \\
\hline QRS duration & $0.53[0.48,0.58]$ & $0.53[0.40,0.66]$ & $0.26[0.04,0.48]$ & $0.23[0.00,0.46]$ \\
\hline QTc interval ${ }^{a}$ & $0.60[0.55,0.65]$ & $0.55[0.42,0.68]$ & $0.30[0.08,0.52]$ & $0.28[0.05,0.52]$ \\
\hline $\mathrm{RV} 5+\mathrm{SV} 1^{\mathrm{b}}$ & $0.68[0.63,0.72]$ & $0.55[0.40,0.70]$ & $0.00[-0.33,0.33]$ & $0.00[-0.34,0.34]$ \\
\hline SL product ${ }^{\mathrm{C}}$ & $0.61[0.54,0.67]$ & $0.51[0.36,0.66]$ & $0.04[-0.21,0.29]$ & $0.05[-0.20,0.31]$ \\
\hline C product ${ }^{d}$ & $0.47[0.39,0.55]$ & $0.67[0.53,0.81]$ & $0.01[-0.24,0.26]$ & $0.00[-0.26,0.26]$ \\
\hline $\mathrm{BMI}^{\mathrm{e}}$ & $0.76[0.73,0.80]$ & $0.79[0.68,0.91]$ & $0.14[-0.11,0.39]$ & $0.16[-0.09,0.41]$ \\
\hline Height & $0.88[0.86,0.89]$ & $1.00[0.90,1.10]$ & $0.40[0.18,0.63]$ & $0.39[0.17,0.61]$ \\
\hline
\end{tabular}

Note: The resting ECG variables were corrected for age and ECG machine and five principal components were used as covariates. Significant heritability estimates are indicated in bold type. $\mathrm{Cl}=$ confidence interval; SL product = Sokolow-Lyon product; $\mathrm{C}$ product = Cornell product; $\mathrm{BMI}$ $=$ body mass index. ${ }^{\mathrm{a}} \mathrm{QT}$ interval additionally corrected for RR interval; ${ }^{\mathrm{b}} \mathrm{n}=1,967$ for the estimates including both $\mathrm{MZ}$ twins $/ 1,625$ for the estimates including only the single genotyped $M Z$ twins; ${ }^{c} n=2,330 / 2,091 ;{ }^{d} n=2,317 / 2,078 ;{ }^{e} B M I$ was log-transformed, $n=2,531 / 2,114$.

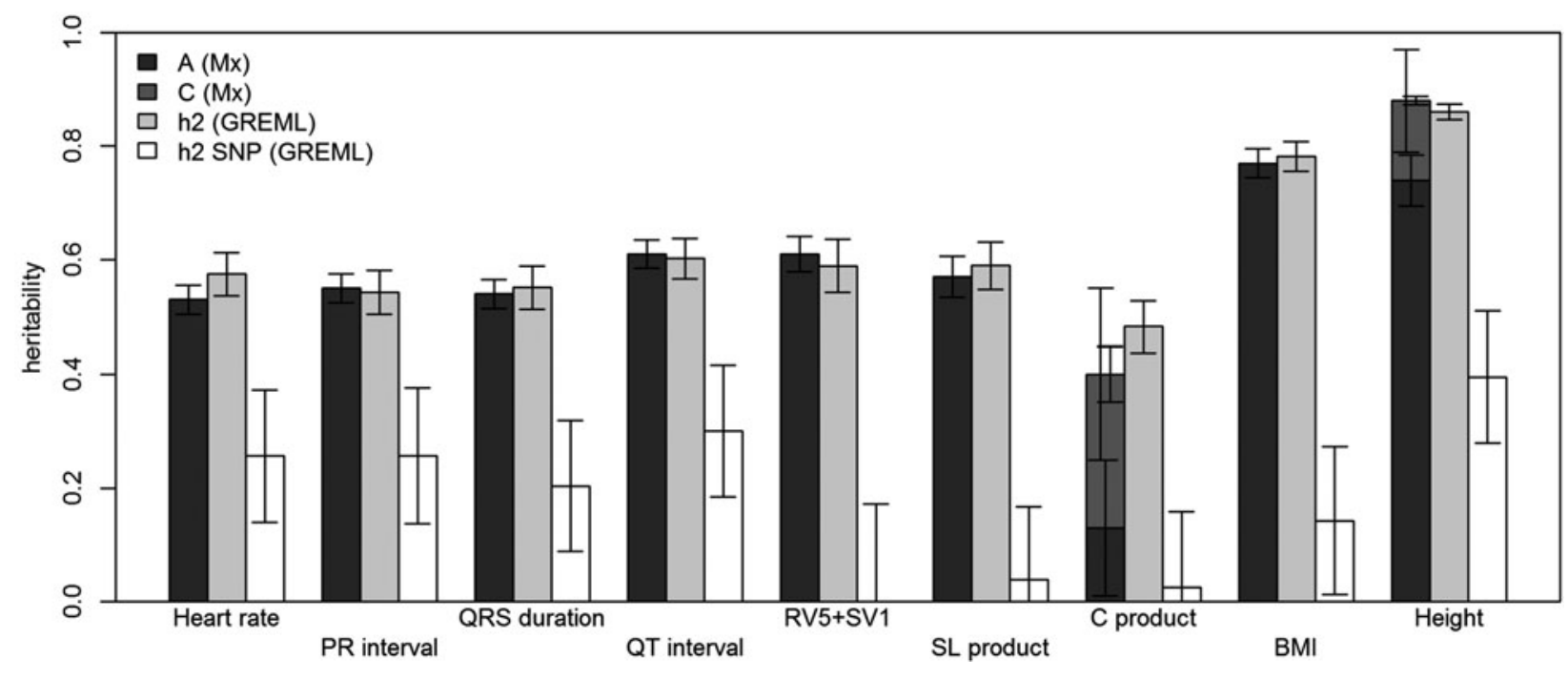

\section{FIGURE 1}

Comparison of heritability estimates by Mx and GREML using both MZ twins. Left bars show narrow-sense heritability estimates (A) stacked with the estimates of shared environment (C) from $M x$, if these were included in the best fitting model; the grey bars in the middle show the narrow-sense heritability estimates from GREML, and the white bars show the common SNP heritability estimates from GREML. Error bars indicate the standard errors of the estimates. For the traits for which $\mathrm{C}$ was significant, the left error bars indicate the standard errors of $\mathrm{C}$ and the right ones those for $\mathrm{A}+\mathrm{C}$.

Note: SL product $=$ Sokolow-Lyon product; $\mathrm{C}$ product $=$ Cornell product.

all studies on heart function traits were consistent with a model where trait resemblance was solely due to additive genetic factors; that is, there was no evidence for a contribution of shared environmental factors (Polderman et al., 2015).

We are the first to analyze these ECG traits in the same cohort using both twin modeling and GREML analysis, which provided us with the unique opportunity to compare the results of these methods. GREML analysis estimates $h^{2}$ without assumptions on genetic similarity and provides unbiased estimates in the case of violation of the equal environment assumption when only one of the MZ twins is used, as shown in the small simulation study. MZ twins might share more of their prenatal environmental in- fluences (e.g., through a shared placenta) than DZ twins, which might have caused a more similar early development of the cardiac conduction system, but such a scenario was not supported by our data. As the classical twin model and GREML using both MZ twins did not yield higher $h^{2}$ estimates than GREML using only the genotyped $M Z$ twin, it is unlikely that the equal environment assumption for MZ and DZ twins was violated and hence the $h^{2}$ estimates are likely not inflated for at least ECG traits, but potentially also for other complex traits. Note that the violation of the equal environment assumption can also bias the $h^{2}$ estimates when shared environment is not included in the best fitting model as is the case for all but one of the ECG traits. If MZ twins share their environments to a greater extent than $\mathrm{DZ}$ twins, 


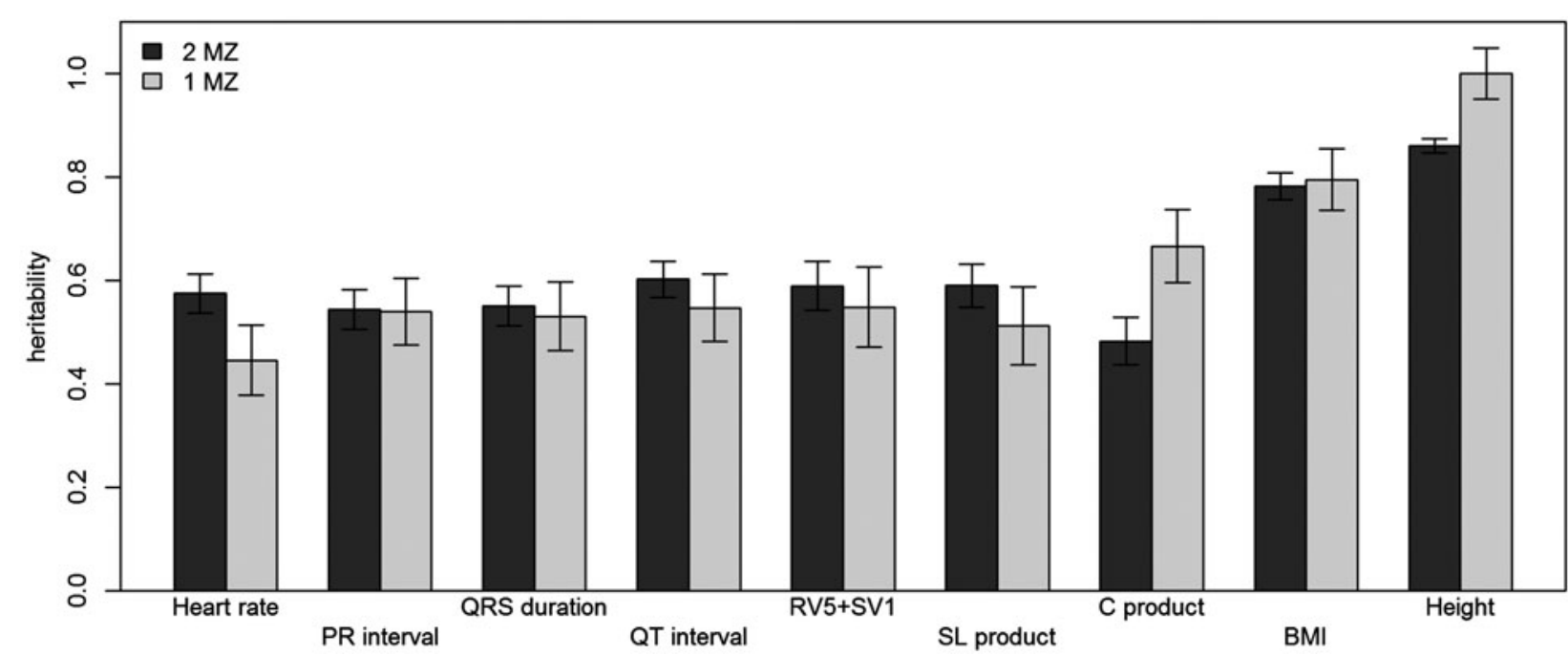

\section{FIGURE 2}

Comparison of narrow-sense heritability estimates by GREML using both MZ twins with those when only the genotyped MZ twin was included. Dark gray bars show the heritability estimates from GREML using both MZ twins and light gray bars show those using only the genotyped $\mathrm{MZ}$ twin. Error bars indicate the standard errors of the estimates.

Note: SL product $=$ Sokolow-Lyon product; C product $=$ Cornell product.

such increased environmental sharing in MZ pairs affecting ECG traits will be incorrectly attributed to A and yield an inflated $h^{2}$.

The common SNP heritability estimates were only statistically significant for heart rate, PR-interval, QRS duration, and QTc-interval, and were roughly half of the $h^{2}$ values for these traits. This suggests that common genetic factors account for a substantial part of the phenotypic variation in ECG traits. However, currently identified variants for these traits constitute only a small fraction of these $h^{2}$ SNP estimates, implying that many more variants may be discovered through larger meta-GWAS studies. The remaining $h^{2}$ is likely due to other types of genetic variation, including rare variants and copy number variations. Such variants could in the future be identified using exome chip or whole genome sequencing studies. The lack of statistically significant $h^{2}$ SNP values for the other traits may partly be caused by the lower sample sizes that were available for analysis of these traits.

Concerning the common SNP heritability, it was interesting to see that the estimates of $h^{2}{ }_{\text {IBS }>0.05}$ and $h^{2}$ SNP by joint modeling using GREML yielded more accurate $h^{2}$ SNP estimates than by applying GREML to unrelated individuals only. We would therefore advise future studies aiming to estimate the common SNP heritability to use all genetic data available and not to limit the study to only unrelated individuals. With the recently proposed GREMLLDMS method, only one $h^{2}$ SNP estimate was significant, but it was very inaccurate due to the large standard error. GREML-LDMS was actually developed for application to next-generation sequence or densely imputed genotype data, in order to yield an estimate of the narrow-sense her- itability when applied to unrelated individuals as such data include both rare variants and common ones, that is, all variation in the genome. However, our genotype data set only included common variants and hence could only provide an estimate for the common SNP heritability. Recently, Nivard et al. (2016) extended Zaitlen's method to determine moderator effects on both the total and the common SNP heritability. It would be interesting to determine in future research whether the heritability of cardiac conduction outcomes changes with, for example, age or body mass index (BMI).

As a proof of principle we also applied the same methods to BMI and height, two model complex traits that have been widely studied by others. For BMI and height, the $h^{2}$ estimates by $\mathrm{Mx}$ were 0.75 and 0.74 , respectively, which is comparable to estimates observed in other twin studies (Elks et al., 2012; Polderman et al., 2015). Additionally, 14\% of variability in height could be explained by shared environment. For BMI comparable estimates were found with GREML $\left(h^{2}=h^{2}=0.76\right)$, while for height the GREML estimates were 0.88 . This latter estimate is close to the (familial) effect of $\mathrm{A}$ and $\mathrm{C}$ combined found by $\mathrm{Mx}$. A similar result was observed for the Cornell product, which was the only ECG trait for which Mx included the C in the best fitting model. Zaitlen and colleagues already showed that GREML analysis using sibs resulted in larger $h^{2}$ estimates than when using more distant relatives, suggesting that the GREML $h^{2}$ estimates might be confounded by shared environment (Zaitlen et al., 2013). A recent review exploring BMI $h^{2}$ estimates from different study designs came to the same conclusion (Robinson et al., 2017). Therefore, the $h^{2}$ 
estimates for height and Cornell product found in this study might be inflated because of confounding by shared environment. However, the Mx estimates of $\mathrm{C}$ might not entirely reflect true effects of shared environment, as it is well known that the classical twin design often lacks power to clearly discriminate between A and C (Keller et al., 2010). At any rate, our results indicate that in those cases where a significant $\mathrm{C}$ is detected, the $\mathrm{Mx}$ estimate of the classical twin study may underestimate rather than overestimate $h^{2}$.

A limitation of our study might be that the sample used for the GREML estimation is not exactly the same as the one used for classical twin modeling. GWAS data were not available for all twins who had phenotype data. On the other hand, some singletons did have GWAS and phenotype data and could therefore be included in the GREML analyses. Nevertheless, the sample size did not differ much, so we believe that the heritability estimates can validly be compared between methods. A second limitation is that the study sample contained predominantly middle-aged females. It might be that the heritability of cardiac conduction traits changes over time and that our heritability estimates are different from those in the general population. Our results can therefore not be generalized. Third, at baseline we found a statistically significant difference between the groups of $\mathrm{MZ}$ and $\mathrm{DZ}$ twins for QRS duration. As this difference was biologically small, it is likely due to the large sample size and does not represent an important biological difference between the groups. There were no statistically significant differences in the prevalence of atrial fibrillation, AV-block, LVH, T-axis orientation, or bundle branch blocks. The number of affected cases was too low to reliably estimate $h^{2}$ on these diagnostic ECG categories using GREML.

In summary, our data indicate that genetic factors play an important role in determining the variation in heart rate, PR interval, QRS duration, QTc interval, RV5+SV1, Sokolow-Lyon voltage, and possibly Cornell voltage. Furthermore, we found that estimates from the classical twin model can be relied on for determining heritability of ECG traits and that these were not inflated due to increased environmental sharing between $\mathrm{MZ}$ compared to $\mathrm{DZ}$ twin pairs. Together, our results and those of others provide a solid basis for efforts attempting to identify genetic variants responsible for the heritabilities of the ECG parameters in the general population. Identification of a growing number of genetic associations to electrocardiographic parameters and outcome variables such as sudden cardiac death, which can be reliably and reproducibly identified, will add considerably to future scientific, therapeutic, and preventive options regarding cardiovascular diseases.

\section{Acknowledgments}

TwinsUK: The study was supported by the Wellcome Trust; European Community's Seventh Framework Programme
(FP7/2007-2013). The study also receives support from the National Institute for Health Research (NIHR)-funded BioResource, Clinical Research Facility and Biomedical Research Centre based at Guy's and St Thomas' NHS Foundation Trust in partnership with King's College London. SNP genotyping was performed by The Wellcome Trust Sanger Institute and National Eye Institute via NIH/CIDR.

\section{Conflicts of Interest}

None.

\section{Supplementary Material}

To view supplementary material for this article, please visit https://doi.org/10.1017/thg.2017.55.

\section{References}

Algra, A., Tijssen, J. G., Roelandt, J. R., Pool, J., \& Lubsen, J. (1991). QTc prolongation measured by standard 12-lead electrocardiography is an independent risk factor for sudden death due to cardiac arrest. Circulation, 83, 1888-1894.

Andrew, T., Hart, D. J., Snieder, H., de Lange, M., Spector, T. D., \& MacGregor, A. J. (2001). Are twins and singletons comparable? A study of disease-related and lifestyle characteristics in adult women. Twin Research, 4, 464-477.

Arking, D. E., Pfeufer, A., Post, W., Kao, W. H., Newton-Cheh, C., Ikeda, M., ... Chakravarti, A. (2006). A common genetic variant in the NOS1 regulator NOS1AP modulates cardiac repolarization. Nature Genetics, 38, 644-651.

Calderón, A., Barrios, V., Escobar, C., Ferrer, E., Barrios, S., González-Pedel, V., ... Navarro-Cid, J. (2010). Detection of left ventricular hypertrophy by different electrocardiographic criteria in clinical practice. Findings from the Sara study. Clinical and Experimental Hypertension, 32, 145153.

Chambers, J. C., Zhao, J., Terracciano, C. M. N., Bezzina, C. R., Zhang, W., Kaba, R., ... Kooner, J. S. (2010). Genetic variation in SCN10A influences cardiac conduction. Nature Genetics, 42, 149-152.

Chang, K.-C., Barth, A. S., Sasano, T., Kizana, E., Kashiwakura, Y., Zhang, Y., ... Marbán, E. (2008). CAPON modulates cardiac repolarization via neuronal nitric oxide synthase signaling in the heart. Proceedings of the National Academy of Sciences of the United States of America, 105, 4477-4482.

Copie, X., Hnatkova, K., Staunton, A., Fei, L., Camm, A. J., \& Malik, M. (1996). Predictive power of increased heart rate versus depressed left ventricular ejection fraction and heart rate variability for risk stratification after myocardial infarction. Results of a two-year follow-up study. Journal of the American College of Cardiology, 27, 270-276.

Dalageorgou, C., Ge, D., Jamshidi, Y., Nolte, I. M., Riese, H., Savelieva, I., ... Snieder, H. (2008). Heritability of QT interval: How much is explained by genes for resting heart rate? Journal of Cardiovascular Electrophysiology, 19, 386-391.

Dekker, J. M., Crow, R. S., Hannan, P. J., Schouten, E. G., \& Folsom, A. R. (2004). Heart rate-corrected QT interval prolongation predicts risk of coronary heart disease in black 
and white middle-aged men and women: The ARIC study. Journal of the American College of Cardiology, 43, 565-571.

Dekker, J. M., Schouten, E. G., Klootwijk, P., Pool, J., \& Kromhout, D. (1994). Association between QT interval and coronary heart disease in middle-aged and elderly men. The Zutphen Study. Circulation, 90, 779-785.

Elks, C. E, den Hoed, M., Zhao, J. H., Sharp, S. J., Wareham, N. J., Loos, R. J., \& Ong, K. K. (2012).Variability in the heritability of body mass index: A systematic review and metaregression. Frontiers int Endocrinology (Lausanne), 3, 29.

George, A. L., Crotti, L., Monti, M. C., Peljto, A., Goosen, A., Brink, P. A., ... George, A. L. Jr. (2009). NOS1AP is a genetic modifier of the long-QT syndrome. Circulation, 120, 16571663.

Hanson, B., Tuna, N., Bouchard, T., Heston, L., Eckert, E., Lykken, D., ... Rich, S. (1989). Genetic factors in the electrocardiogram and heart rate of twins reared apart and together. American Journal of Cardiology, 63, 606-609.

Havlik, R. J., Garrison, R. J., Fabsitz, R., \& Feinleib, M. (1980). Variability of heart rate, P-R, QRS and Q-T durations in twins. Journal of Electrocardiology, 13, 45-48.

Holm, H., Gudbjartsson, D. F., Arnar, D. O., Thorleifsson, G., Thorgeirsson, G., Stefansdottir, H., ... Stefansson, K. (2010) Several common variants modulate heart rate, PR interval and QRS duration. Nature Genetics, 42, 117-122.

Hong, Y., Rautaharju, P. M., Hopkins, P. N., Arnett, D. K., Djoussé, L., Pankow, J. S., ... Province, M. A. (2001). Familial aggregation of QT-interval variability in a general population: Results from the NHLBI Family Heart Study. Clinical Genetics, 59, 171-177.

Howie, B. N., Donnelly, P., \& Marchini, J. (2009). A flexible and accurate genotype imputation method for the next generation of genome-wide association studies. PLoS Genetics, 5, e1000529.

International HapMap 3 Consortium. (2010). Integrating common and rare genetic variation in diverse human populations. Nature, 467, 52-58.

Keller, M. C., Medland, S. E., \& Duncan, L. E. (2010). Are extended twin family designs worth the trouble? A comparison of the bias, precision, and accuracy of parameters estimated in four twin family models. Behavioral Genetics, 3, 377-393.

Li, J., Huo, Y., \& Zhang, Y. (2009). Familial aggregation and heritability of electrocardiographic intervals and heart rate in a rural Chinese population. Annals of Noninvasive Electrocardiology, 14, 147-152.

Maher, B. (2008). Personal genomes: The case of the missing heritability. Nature, 456, 18-21.

Mathers, J. A., Osborne, R. H., \& DeGeorge, F. V. (1961). Studies of blood pressure, heart rate, and the electrocardiogram in adult twins. American Heart Journal, 62, 634-642.

Moayyeri, A., Hammond, C., Hart, D., \& Spector, T. (2013). The UK adult twin registry (TwinsUK Resource). Twin Research and Human Genetics, 16, 144-149.

Murkofsky, R. L., Dangas, G., Diamond, J. A., Mehta, D., Schaffer, A., \& Ambrose, J. A. (1998). A prolonged QRS duration on surface electrocardiogram is a specific indicator of left ventricular dysfunction. Journal of the American College of Cardiology, 32, 476-482.

Mutikainen, S., Ortega-Alonso, A., Alén, M., Kaprio, J., Karjalainen, J., Rantanen, T., \& Kujala, U. M. (2009). Genetic influences on resting electrocardiographic variables in older women: A twin study. Annals of Noninvasive Electrocardiology, 14, 57-64.

Neale, M., Boker, S., Xie, G., \& Maes, H. (2003). Mx: statistical modeling (6th ed.). Richmond, VA: Department of Psychiatry.

Newton-Cheh, C., Eijgelsheim, M., Rice, K. M., de Bakker, P. I., Yin, X., Estrada, K., ... Stricker, B. H. (2009). Common variants at ten loci influence QT interval duration in the QTGEN Study. Nature Genetics, 41, 399-406.

Newton-Cheh, C., Guo, C.-Y., Wang, T. J., O’Donnell, C. J., Levy, D., \& Larson, M. G. (2007). Genome-wide association study of electrocardiographic and heart rate variability traits: The Framingham Heart Study. BMC Medical Genetics, $8, \mathrm{~S} 2$.

Nivard, M. G., Middeldorp, C. M., Lubke, G., Hottenga, J. J., Abdellaoui, A., Boomsma, D. I., \& Dolan, C. V. (2016). Detection of gene-environment interaction in pedigree data using genome-wide genotypes. European Journal of Human Genetics, 2, 1803-1809.

Nolte, I. M., Wallace, C., Newhouse, S. J., Waggott, D., Fu, J., Soranzo, N., ... Jamshidi, Y. (2009). Common genetic variation near the phospholamban gene is associated with cardiac repolarisation: Meta-analysis of three genome-wide association studies. PLoS One, 4, e6138.

Pfeufer, A., Sanna, S., Arking, D. E., Müller, M., Gateva, V., Fuchsberger, C., ... Chakravarti, A. (2009). Common variants at ten loci modulate the QT interval duration in the QTSCD Study. Nature Genetics, 41, 407-414.

Pfeufer, A., van Noord, C., Marciante, K. D., Arking, D. E., Larson, M. G., Smith, A. V., ... Heckbert, S. R. (2010). Genome-wide association study of PR interval. Nature Genetics, 42, 153-159.

Polderman, T. J. C., Benyamin, B., de Leeuw, C. A., Sullivan, P. F., van Bochoven, A., Visscher, P. M., \& Posthuma, D. (2015). Meta-analysis of the heritability of human traits based on fifty years of twin studies. Nature Genetics, 47, 702-709.

Purcell, S., Neale, B., Todd-Brown, K., Thomas, L., Ferreira, M. A., Bender, D., ... Sham, P. C. (2007). PLINK: A tool set for whole-genome association and population-based linkage analyses. American Journal of Human Genetics, 81, 559575.

Robinson, M. R., English, G., Moser, G., Lloyd-Jones, L. R., Triplett, M. A., Zhu, Z., ... Visscher, P. M. (2017). Genotypecovariate interaction effects and the heritability of adult body mass index. Nature Genetics, 49, 1174-1181.

Russell, M. W., Law, I., Sholinsky, P., \& Fabsitz, R. R. (1998). Heritability of ECG measurements in adult male twins. Journal of Electrocardiology, 30, 64-68.

Schouten, E. G., Dekker, J. M., Meppelink, P., Kok, F. J., Vandenbroucke, J. P., \& Pool, J. (1991). QT interval prolongation predicts cardiovascular mortality in an apparently healthy population. Circulation, 84, 1516-1523. 
Singh, J. P., Larson, M. G., O’Donnell, C. J., Tsuji, H., Evans, J. C., \& Levy, D. (1999). Heritability of heart rate variability: The Framingham Heart Study. Circulation, 99, 2251-2254.

Smith, J. G., Lowe, J. K., Kovvali, S., Maller, J. B., Salit, J., Daly, M. J., ... Newton-Cheh, C. (2009). Genome-wide association study of electrocardiographic conduction measures in an isolated founder population: Kosrae. Heart Rhythm, 6, 634-641.

Storstein, L., Bjørnstad, H., Hals, O., \& Meen, H. D. (1991). Electrocardiographic findings according to sex in athletes and controls. Cardiology, 79, 227-236.

Turkheimer, E. (2011). Still missing. Research in Human Development, 8, 227-241.

Visscher, P. M., Medland, S. E., Ferreira, M. A. R., Morley, K. I., Zhu, G., Cornes, B. K., ... Martin, N. G. (2006). Assumption-free estimation of heritability from genomewide identity-by-descent sharing between full siblings. PLoS Genetics, 2, 316-325.

Voss, A., Busjahn, A., Wessel, N., Schurath, R., Faulhaber, H. D., Luft, F. C., \& Dietz, R. (1996). Familial and genetic influences on heart rate variability. Journal of Electrocardiology, 29, 154-160.

Yang, J., Bakshi, A., Zhu, Z., Hemani, G., Vinkhuyzen, A. A., Lee, S.H., ... Visscher, P. M. (2015). Genetic variance esti- mation with imputed variants finds negligible missing heritability for human height and body mass index. Nature Genetics, 47, 1114-1120.

Yang, J., Benyamin, B., McEvoy, B., Gordon, S., Henders, A. K., Nyholt, D. R., ... Visscher, P. M. (2010). Common SNPs explain a large proportion of the heritability for human height. Nature Genetics, 42, 565-571.

Yang, J., Lee, S. H., Goddard, M. E., \& Visscher, P. M. (2011). GCTA: A tool for genome-wide complex trait analysis. American Journal of Human Genetics, 88, 76-82.

Yasumura, S., \& Shibata, H. (1989). The effect of aging on the electrocardiographic findings in the elderly: A 10-year longitudinal study: The Koganei Study. Archives of Gerontology and Geriatrics, 9, 1-15.

Zaitlen, N., Kraft, P., Patterson, N., Pasaniuc, B., Bhatia, G., Pollack, S., \& Price, A. L. (2013). Using extended genealogy to estimate components of heritability for 23 quantitative and dichotomous traits. PLoS Genetics, 9, e1003520.

Zuk, O., Hechter, E., Sunyaev, S. R., \& Lander, E. S. (2012). The mystery of missing heritability: Genetic interactions create phantom heritability. Proceedings of the National Academy of Sciences of the United States of America, 109, 1193-1198. 